Caja Núñez, C., Cascales Martínez, A. \& Gomariz Vicente, M.A. (2017). Valoración desde la perspectiva de los orientadores de Educación Secundaria de la Región de Murcia del portal de Atención a la Diversidad ORIENTAMUR. Revista Electrónica Interuniversitaria de Formación del Profesorado, 20(1), 49-68.

\title{
Valoración desde la perspectiva de los orientadores de Educación Secundaria de la Región de Murcia del portal de Atención a la Diversidad ORIENTAMUR.
}

\author{
Cristel Caja Núñez, Antonia Cascales Martínez, Mª Ángeles Gomariz Vicente
}

Universidad de Murcia. Facultad de Educación. Departamento de Métodos de Investigación y Diagnóstico en Educación.

\section{Resumen}

En el presente trabajo se pretende dar a conocer las opiniones que albergan los orientadores de Educación Secundaria sobre la adecuación y utilidad de los recursos ofertados por la Consejería de Educación y Universidades de la Comunidad Autónoma de la Región de Murcia, en materia de atención a la diversidad, a los centros educativos de Educación Secundaria de esta región, desde el portal de Atención a la Diversidad: ORIENTAMUR.

Es un estudio principalmente de carácter descriptivo realizado a través de una encuesta a orientadores de secundaria que han querido colaborar en este proyecto.

Los resultados nos muestran que existe una satisfacción general en torno a este portal, especialmente en cuanto a su disposición web, manejo y funcionamiento. Sin embargo, algunos aspectos relacionados con los recursos disponibles podrían perfeccionarse haciendo que esta página web sea y continúe siendo un referente para todos los orientadores de esta región.

\section{Palabras clave}

Recursos; orientación educativa; atención a la diversidad; web educativa.

Contacto:

Cristel Caja Núñez, cristel.caja@um.es. Facultad de Educación - Universidad de Murcia 


\title{
Assessment from the perspective of practitioners of Secondary Education of the Region of Murcia of ORIENTAMUR Care Diversity portal.
}

\begin{abstract}
The present project aims to show the opinions of Secondary School counselors about the adequacy and usefulness of the resources offered by the Ministry of Education, Culture and Universities of the Region of Murcia, in providing the necessary care and attention to diversity in the Secondary Schools in this region, through the portal of Attention to Diversity: ORIENTAMUR.

It is a descriptive study, conducted mainly through a survey of Secondary School counselors who have agreed to collaborate on this project.

The results show that there is general level of satisfaction with this website, especially in the web layout, management and how easy it is to use. However, some aspects of the available resources could be improved, in this way this webpage would become and remain a useful and essential reference for all school counselors in this Region.
\end{abstract}

\section{Keywords}

Resources; educational orientation; attention to diversity; educational website.

\section{Introducción}

En la actualidad las Tecnologías de la Información y la Comunicación tienen una presencia consolidada en el campo de la educación. En este contexto, los portales educativos dependientes de las administraciones educativas se han convertido en el tablón de anuncios por excelencia. Un portal educativo se define como un sitio web en el que se incluyen tanto contenidos como servicios y que se brindan a la comunidad educativa en su conjunto (docentes, alumnado, familias, etc.).

En esta línea, Marqués (1998) nos indica que los buenos espacios formativos web son eficaces, facilitan el logro de sus objetivos y ello es debido a una serie de características que atienden a diversos aspectos funcionales, técnicos, estéticos psicológicos y pedagógicos.

Desde una perspectiva más general, también centrada en la elaboración de instrumentos fiables y validos que permitan evaluar la calidad de un programa y recursos educativos, Arnáiz y Guirao (2015) nos presentan el proceso de diseño, depuración y validación del instrumento ACADI (Autoevaluación de Centros para la Atención a la Diversidad desde la Inclusión) creando un instrumento válido y fiable para la autoevaluación de centros educativos de Infantil, Primaria y Secundaria; utilizando una investigación similar a la nuestra. Marcelo y Zapata (2008) nos aportan diferentes dimensiones para la evaluación de la calidad para programas completos de formación docente. En este caso, apuestan por evaluar el contexto, el diseño, producción, puesta en marcha y seguimiento. Cabero y Duarte (2012) evalúan medios y materiales de enseñanza en soporte multimedia a través de diferentes dimensiones como: calidad instructiva en general, contenido, adecuación del programa, técnicas de formulación de preguntas, enfoque/motivación, control del estudiante, objetivos de aprendizaje.... Por último, mencionar a Mirete, Cabello, Martínez- 
Segura y García-Sánchez (2010), grupo formado de Investigación, Educación y Calidad de la Universidad de Murcia que crean un cuestionario para la evaluación de aspectos didácticos, técnicos y pedagógicos de web didácticas. La combinación de todas estas características permite crear una página web, portal educativo, soporte multimedia... de calidad.

La Ley Orgánica 2/2006, de 3 de mayo de Educación (LOE), en su artículo 79 nos indica que "El Ministerio de Educación, Cultura y Deporte ofrecerá plataformas digitales y tecnológicas de acceso a toda la comunidad educativa, que podrán incorporar recursos didácticos aportados por las Administraciones educativas y otros agentes para su uso compartido".

Es por ello la decisión de acercarnos al portal de la Región de Murcia ORIENTAMUR y analizar sus recursos para la atención a la diversidad siendo éste, como ya hemos planteado un tema de plena actualidad.

ORIENTAMUR se creó en el año 2009, alojado en el dominio de murciaeduca.es, entrando en funcionamiento ese mismo año. El portal fue impulsado por la Consejería de Educación, Cultura y Universidades, y en concreto por la Dirección General de Promoción, Ordenación e Innovación Educativa. Actualmente alberga más de 500 documentos. Este portal, configurado para la atención a la diversidad, afirma que la Comunidad Autónoma de la Región de Murcia asume el reto de una educación en igualdad de oportunidades que atienda a todo el alumnado, buscando una respuesta adecuada a sus características y necesidades, y que aborda, además, los grupos de alumnos y alumnas que requieren una atención educativa diferente a la ordinaria, es decir, respuestas para la atención a la diversidad.

La Ley Orgánica 8/2013, de 9 de diciembre, para la Mejora de la Calidad Educativa (LOMCE), reafirmando lo ya promulgado por la LOE en su artículo 22.4 señala la atención a la diversidad, además de la educación común, como un principio general fundamental en la Educación Secundaria Obligatoria. También señala, como principio del alumnado con necesidad específica de apoyo educativo, en su artículo 71.1, que las Administraciones educativas dispondrán los medios necesarios para que todo el alumnado alcance el máximo desarrollo personal, intelectual, social y emocional, así como los objetivos establecidos con carácter general en la presente Ley. Continúa añadiendo que corresponde a las Administraciones educativas asegurar los recursos necesarios para que los alumnos y alumnas que requieran una atención educativa diferente a la ordinaria, puedan alcanzar el máximo desarrollo posible de sus capacidades personales y, en todo caso, los objetivos establecidos con carácter general para todo el alumnado. En definitiva, corresponde a las Administraciones educativas dotar a los centros de los recursos necesarios para atender adecuadamente a cualquier alumnado que lo necesite por diferentes causas, en nuestro caso, será la Consejería de Educación, Cultura y Universidades de la CARM la encargada de proporcionar esas medidas y recursos para la atención a la diversidad. Estos recursos serán principalmente administrados a través del portal ORIENTAMUR. Este portal educativo es un repositorio, en él encontramos fundamentalmente documentación, normativa y enlaces de interés organizados por diferentes dimensiones.

Las investigaciones en torno a esta temática son escasas. Cano, Mayoral, Liesa y Castelló $(2008,2011)$ realizan una valoración de las funciones del profesor de orientación educativa en Cataluña formando parte de un proyecto de investigación fundamental. En dicho estudio, analizan el nivel de satisfacción y de obstáculos con los que se encuentra el profesor de la especialidad de orientación educativa en Cataluña.

La literatura respecto a los obstáculos percibidos en la función asesora del orientador es abundante (Aciego de Mendoza y Muñoz 2005; Álvarez, Bethecourt y Cabrera, 2000; Comes, Parera, Vedriel y Vives, 2009; Liesa, Castelló, Carretero, Cano y Mayoral, 2012; Vélaz 
de Medrano, 2001; Vélaz de Medrano et al., 2012) nos muestra que existen seis grandes obstáculos o dificultades principales: el tiempo, la coordinación y colaboración entre profesionales, las demandas y necesidades del alumnado, la atención a la diversidad, el trabajo con las familias y la falta de recursos (Cano et al., 2013). Carballo, Díaz, Fernández y García (1997) nos reiteran la escasez de recursos, entre otros factores, en su estudio de investigación sobre "Orientación en Educación Secundaria. Situación actual y prospectiva"

Por último, una de las conclusiones extraídas en el trabajo de Escarbajal et al.(2012) apunta que es fundamental, de cara a la generación de contextos educativos más inclusivos, prestar una especial atención a aspectos como la formación del profesorado, las condiciones bajo las que se desarrolla, el número de profesores por aula y los apoyos con los que cuenta, así como su organización, aplicación de medidas específicas, entre otros.

Nos encontramos en una situación cambio legislativo es necesario dotar a los orientadores de recursos, tanto normativa como instrucciones para abordar esta nueva etapa. Por ello, la tutela por parte de la Consejería de Educación es esencial para poder, con esta nueva reforma, conducir a nuestro sistema educativo a una notable mejora, tal y como la LOMCE afirma.

El objetivo general que se pretende con esta investigación es determinar la adecuación y utilidad de los recursos ofertados por la Consejería de Educación, Cultura y Universidades de la Comunidad Autónoma de la Región de Murcia, a través de ORIENTAMUR, a los centros educativos de Educación Secundaria de esta Región, desde la perspectiva de los orientadores de esta etapa educativa.

Este propósito se concreta en dos objetivos específicos:

1. Conocer la opinión de los orientadores, respecto a dicho portal.

2. Analizar la influencia de las variables sociodemográficas en la valoración que del portal ORIENTAMUR hacen los orientadores.

\section{Metodología}

Presentamos un estudio que adopta un planteamiento descriptivo con diseño de grupo único, cuya finalidad exploratoria trata de determinarla adecuación y utilidad del portal educativo ORIENTAMUR según los orientadores.

Los participantes en esta investigación son 45 orientadores que desempeñan su labor en centros de Educación Secundaria de diversa titularidad existentes en esta Comunidad Autónoma, lo que supone un $17,3 \%$ de la totalidad de los orientadores de la Región de Murcia. La selección ha respondido a un muestreo intencional respetando el procedimiento de grupo intacto. En cuanto al perfil profesional, un $55 \%$ son psicólogos, un $35 \%$ pedagogos y un $10 \%$ psicopedagogos entre los cuales destaca notablemente el sexo femenino $(77,8 \%)$ frente al masculino. Un $66,7 \%$ de los encuestados considera su formación en TIC es a nivel de usuario y solo un $9 \%$ considera que su nivel es básico. Se ha utilizado un diseño descriptivo propio de un estudio tipo encuesta (Hernández y Maquilón, 2010). A fin de determinar las relaciones existentes entre las diferentes variables implicadas, un objetivo característico de esta modalidad de investigación, las variables predictoras consideradas han sido: titulación, sexo, edad, experiencia laboral, grado de formación en TIC y situación laboral. Por su parte, las variables criterio que se han estimado son: accesibilidad al portal de Atención a la Diversidad ORIENTAMUR, usabilidad/aspectos técnicos, organización y presentación de los recursos, utilidad/adecuación de los recursos, aspectos pedagógicos y motivación e interés de los recursos. El análisis cuantitativo de la información se ha 
realizado con el paquete estadístico IBM SPSS Statistic (vers. 19) y ha consistido en el cálculo de estadísticos descriptivos, Alfa de Cronbach para comprobar la fiabilidad del instrumento $\mathrm{y}$, para el contraste de medias, las pruebas de estadística no paramétrica: prueba de Kruskal-Wallis y prueba $U$ de Mann Whitney.

La información para dar respuesta a los objetivos de este trabajo se ha recogido mediante un cuestionario on-line anónimo e individual diseñado ad hoc, a través de la aplicación encuestas que ofrece la Universidad de Murcia. La propia naturaleza del instrumento que parte de las inquietudes de los autores tras consultar la bibliografía estudiada aconsejaba su validación con una estrategia de fiabilidad entre jueces de carácter cualitativo, para lo cual se contó con tres expertos: un orientador de un Equipo de Orientación Educativa y Psicopedagógica, un doctor en pedagogía y un orientador de un Instituto de Educación Secundaria. Tras dicha validación se realizaron mínimas aportaciones al cuestionario semiestructurado como fueron la incorporación de una respuesta a una de las preguntas cerradas y algunas modificaciones de redacción.

La primera parte del cuestionario contempla seis preguntas cerradas sobre características personales y profesionales (titulación, sexo, edad, experiencia laboral, grado de formación en TIC y situación laboral). La segunda parte, está integrada por seis dimensiones tal cual se recogen en la Tabla 1.

Tabla 1.

Dimensiones evaluadas en el instrumento de recogida de opinión de los orientadores

\begin{tabular}{|l|l|c|}
\hline \multicolumn{1}{|c|}{ Dimensión } & \multicolumn{1}{|c|}{ Descripción } & Ítems \\
\hline $\begin{array}{l}\text { 1.Accesibilidad al } \\
\text { portal de Atención a la } \\
\text { Diversidad: } \\
\text { ORIENTAMUR }\end{array}$ & $\begin{array}{l}\text { Con preguntas tales como en qué medida el orientador tiene } \\
\text { acceso permanente al portal desde el Departamento de } \\
\text { Orientación o si la interfaz de usuario cuenta con opciones de } \\
\text { accesibilidad para personas con discapacidad. }\end{array}$ & 1.1 -1.8 \\
\hline $\begin{array}{l}\text { 2.Usabilidad y } \\
\text { aspectos técnicos }\end{array}$ & $\begin{array}{l}\text { Recoge cuestiones sobre en qué grado la navegación es } \\
\text { sencilla e intuitiva o si los enlaces funcionan correctamente. }\end{array}$ & $2.1-2.12$ \\
\hline $\begin{array}{l}\text { 3.Organización y } \\
\text { presentación de los } \\
\text { recursos. }\end{array}$ & $\begin{array}{l}\text { Donde se valora en qué medida los recursos normativos están } \\
\text { actualizados o si el orientador es informado frecuentemente } \\
\text { sobre los nuevos recursos ofrecidos por la Consejería de } \\
\text { Educación. }\end{array}$ & $3.1-3.9$ \\
\hline $\begin{array}{l}\text { 4.Utilidad y } \\
\text { adecuación de los } \\
\text { recursos. }\end{array}$ & $\begin{array}{l}\text { Podemos mencionar en qué grado los orientadores consultan } \\
\text { habitualmente el portal o si los recursos respetan la } \\
\text { normativa correspondiente a derechos de autor y de } \\
\text { propiedad intelectual. }\end{array}$ & $4.1-4.12$ \\
\hline $\begin{array}{l}\text { 5.Aspectos } \\
\text { pedagógicos. }\end{array}$ & $\begin{array}{l}\text { En este apartado valoraremos algunas aspectos tales como } \\
\text { en qué grado los recursos presentan una información actual e } \\
\text { innovadora o si el portal permite contribuciones por parte de } \\
\text { los orientadores. }\end{array}$ & $5.1-5.4$ \\
\hline $\begin{array}{l}\text { 6.Motivación e interés } \\
\text { de los recursos }\end{array}$ & $\begin{array}{l}\text { En el último apartado conoceremos de qué manera los } \\
\text { recursos son motivadores para el trabajo del orientador o si } \\
\text { éstos son difundidos entres sus compañeros por el mismo. }\end{array}$ & $6.1-6.5$ \\
\hline
\end{tabular}

Las preguntas son de respuesta cerrada tipo Likert, a las que se responde según una escala de menor a mayor grado de acuerdo: 1 totalmente en desacuerdo, 2 en desacuerdo, 3 ni de acuerdo ni en desacuerdo, 4 de acuerdo y 5 totalmente de acuerdo. Seguidamente, 
ofrecemos un espacio donde los orientadores pueden realizar observaciones y/o propuestas de mejora que consideren pertinentes en relación a la evaluación realizada así como una valoración global.

Los cuestionarios se enviaron a todos los orientadores, asegurándoles el anonimato y la confidencialidad de la información, y animándoles a participar debido a la importancia de sus aportaciones para el propósito de la investigación y explicándoles las instrucciones de cumplimentación. Así, para su entrega de forma independiente y autónoma, los participantes dispusieron de dos semanas. Participaron un 17,3\% del total, siendo superior al porcentaje de respuesta en este tipo de encuestas on-line que ronda el 10\% (Couper y Miller, 2008).

En el análisis de la fiabilidad del instrumento hemos obtenido una alta consistencia interna, arrojando un coeficiente de Alfa de Cronbach de .95 siendo, según De Vellis (2003), excelente al obtener una puntuación mayor de .90.

\section{Resultados}

Atendiendo a los objetivos del estudio estructuramos los resultados.

1. Conocer la opinión de los orientadores, respecto a dicho portal.

Preguntamos a los Orientadores su opinión respecto a las siguientes dimensiones:

Accesibilidad al portal de Atención a la Diversidad: ORIENTAMUR

Los resultados de la Tabla 2 muestran que el mayor grado de acuerdo mostrado por los orientadores ha sido en el ítem 1.1 "Tengo acceso permanente desde el Departamento de Orientación" ( $M=4,71)$. Asimismo, podemos observar que la media más baja está en el ítem 1.6 "La interfaz de usuario cuenta con opciones de accesibilidad para personas con discapacidad" ( $M=3,45)$, además este ítem ha obtenido el mínimo más bajo ( $M i ́ n=1)$.

Tabla 2

Accesibilidad al portal de ORIENTAMUR desde la perspectiva de los orientadores. Estadísticos descriptivos

\begin{tabular}{|l|c|c|c|c|}
\hline \multicolumn{1}{|c|}{ Ítems accesibilidad } & M & SD & Mín. & Máx. \\
\hline $\begin{array}{l}\text { 1.1 Tengo acceso permanente desde el } \\
\text { Departamento de Orientación. }\end{array}$ & 4,71 &, 589 & 3 & 5 \\
\hline $\begin{array}{l}\text { 1.2 Se visualiza y es accesible desde cualquier } \\
\text { navegador y equipo. }\end{array}$ & 4,56 &, 693 & 2 & 5 \\
\hline $\begin{array}{l}\text { 1.3 La navegación entre las páginas y dentro de las } \\
\text { mismas se hace con suficiente claridad. }\end{array}$ & 4,51 &, 589 & 3 & 5 \\
\hline $\begin{array}{l}\text { 1.4 Es suficientemente clara la “estructura" de } \\
\text { ORIENTAMUR. }\end{array}$ & 4,33 &, 739 & 2 & 5 \\
\hline $\begin{array}{l}\text { 1.5 La distribución de contenidos y enlaces dentro } \\
\text { de la página principal es acertada. }\end{array}$ & 4,20 &, 815 & 2 & 5 \\
\hline $\begin{array}{l}\text { 1.6 La interfaz de usuario cuenta con opciones de } \\
\text { accesibilidad para personas con discapacidad. }\end{array}$ & 3,45 &, 791 & 1 & 5 \\
\hline 1.7 El diseño web es atractivo. & 3,67 &, 769 & 2 & 5 \\
\hline 1.8 Los colores son adecuados. & 3,82 &, 684 & 2 & 5 \\
\hline 1.9 Es fácil volver a la página de inicio. & 4,29 &, 757 & 1 & 5 \\
\hline
\end{tabular}


En cuanto a la diversidad en las respuestas de los orientadores, parece que el ítem con menor homogeneidad es en el 1.5 "La distribución de contenidos y enlaces dentro de la página principal es acertada." (SD= 815). Los ítems con mayor homogeneidad son por igual el 1.1 "Tengo acceso permanente desde el Departamento de Orientación" y 1.3 "La navegación entre las páginas y dentro de las mismas se hace con suficiente claridad." (SD= ,589).

\section{Usabilidad/aspectos técnicos}

Tal y como muestra la Tabla 3, los orientadores están en mayor grado de acuerdo con el ítem 2.8 "Poseo el conocimiento necesario para manejar los recursos adecuadamente" $(M=$ $4,47)$ y difieren en mayor grado con el ítem 2.11 "Los recursos que proceden de buenas prácticas del profesorado son suficientes" $(M=3,58)$.

Tabla 3.

Usabilidad/aspectos técnicos del portal ORIENTAMUR desde la opinión de los orientadores. Estadística descriptiva

\begin{tabular}{|c|c|c|c|c|}
\hline Ítems usabilidad/aspectos técnicos & $M$ & SD & Mín. & Máx. \\
\hline 2.1 La navegación es sencilla e intuitiva. & 4,16 & ,638 & 2 & 5 \\
\hline $\begin{array}{l}\text { 2.2 Las herramientas y elementos de ayuda a la } \\
\text { navegación básicos que permiten al usuario saber su } \\
\text { ubicación dentro de la Web y movilidad por la } \\
\text { misma, son adecuados. }\end{array}$ & 4,11 & ,682 & 3 & 5 \\
\hline $\begin{array}{l}\text { 2.3 La barra de menú a la que se puede acceder } \\
\text { desde todas las pantallas de contenidos, recoge los } \\
\text { intereses principales de tu labor como orientador. }\end{array}$ & 4,13 & 757 & 2 & 5 \\
\hline $\begin{array}{l}\text { 2.4 El buscador de contenidos es claramente } \\
\text { identificable y accesible desde todas las pantallas. }\end{array}$ & 4,25 &, 576 & 3 & 5 \\
\hline 2.5 Los enlaces funcionan correctamente. & 4,27 & ,688 & 2 & 5 \\
\hline $\begin{array}{l}\text { 2.6 El texto es legible en todo el sitio web (contraste } \\
\text { fondo/fuente , tamaño de letra, ... ) }\end{array}$ & 4,40 &, 580 & 3 & 5 \\
\hline $\begin{array}{l}\text { 2.7 Las imágenes, audios y videos están bien } \\
\text { insertados no existiendo dificultad para su correcta } \\
\text { audición y/o visualización. }\end{array}$ & 3,91 & ,793 & 2 & 5 \\
\hline $\begin{array}{l}\text { 2.8 Poseo el conocimiento necesario para manejar } \\
\text { los recursos adecuadamente. }\end{array}$ & 4,47 & ,625 & 3 & 5 \\
\hline $\begin{array}{l}\text { 2.9 Los recursos digitales son fáciles de aplicar en el } \\
\text { contexto educativo. }\end{array}$ & 4,13 & 726 & 2 & 5 \\
\hline $\begin{array}{l}2.10 \text { Los recursos que proceden de documentos } \\
\text { oficiales están actualizados. }\end{array}$ & 4,00 & ,863 & 1 & 5 \\
\hline $\begin{array}{l}\text { 2.11 Los recursos que proceden de buenas prácticas } \\
\text { del profesorado son suficientes. }\end{array}$ & 3,58 &, 965 & 1 & 5 \\
\hline $\begin{array}{l}\text { 2.12 Los recursos que proceden de buenas prácticas } \\
\text { del profesorado me aportan ideas para mi práctica } \\
\text { diaria. }\end{array}$ & 3,96 & 737 & 2 & 5 \\
\hline
\end{tabular}


En cuanto a la homogeneidad y heterogeneidad de respuestas, la más homogénea la obtiene el ítem 2.4 "El buscador de contenidos es claramente identificable y accesible desde todas las pantallas" $(S D=, 576)$. El ítem con mayor diversidad de respuestas ha sido, con diferencia, el 2.11 "Los recursos que proceden de buenas prácticas del profesorado son suficientes" ( $S D=, 965)$.

Organización y presentación de los recursos

Analizando la Tabla 4, podemos ver que el ítem 3.2 "La organización permite una búsqueda selectiva distinguiendo entre grupos temáticos, etapas, dificultades..." ha sido la afirmación que ha tenido mayor grado de acuerdo $(M=4,27)$ y la que ha obtenido de media una puntuación más baja ha sido el ítem 3.5 "Los recursos prácticos son originales y creativos" $(M=3,73)$.

Tabla 4.

Organización y presentación de los recursos desde la opinión de los orientadores del portal ORIENTAMUR. Estadística descriptiva

\begin{tabular}{|l|c|c|c|c|}
\hline \multicolumn{1}{|c|}{ Ítems organización y presentación de los recursos } & M & SD & Mín. & Máx. \\
\hline $\begin{array}{l}\text { 3.1 Los recursos ofertados aparecen organizados } \\
\text { por categorías o temáticas ajustadas a la situación } \\
\text { actual. }\end{array}$ & 4,13 &, 894 & 1 & 5 \\
\hline $\begin{array}{l}\text { 3.2 La organización permite una búsqueda selectiva } \\
\text { distinguiendo entre grupos temáticos, etapas, } \\
\text { dificultades... }\end{array}$ & 4,27 &, 580 & 3 & 5 \\
\hline $\begin{array}{l}\text { 3.3 Los recursos que se recogen en ORIENTAMUR } \\
\text { son útiles para la práctica diaria. }\end{array}$ & 4,22 &, 765 & 3 & 5 \\
\hline $\begin{array}{l}\text { 3.4 Los recursos son adecuados a la etapa a la que } \\
\text { van destinados. }\end{array}$ & 4,24 &, 712 & 2 & 5 \\
\hline 3.5 Los recursos prácticos son originales y creativos. & 3,73 &, 694 & 2 & 5 \\
\hline $\begin{array}{l}3.6 \text { Dispone de enlaces a otros sitios de la red } \\
\text { relacionados con tu profesión y labores en el centro. }\end{array}$ & 3,82 &, 806 & 2 & 5 \\
\hline $\begin{array}{l}\text { 3.7 Existen recursos y guías que te ayudan en temas } \\
\text { específicos de la orientación. }\end{array}$ & 4,19 &, 727 & 2 & 5 \\
\hline $\begin{array}{l}\text { 3.8 Consulto este portal cada vez que busco } \\
\text { información y/o recursos. }\end{array}$ & 4,09 &, 793 & 2 & 5 \\
\hline $\begin{array}{l}\text { 3.9 Soy informado frecuentemente sobre los nuevos } \\
\text { recursos ofrecidos por la Consejería de Educación. }\end{array}$ & 3,91 &, 900 & 1 & 5 \\
\hline
\end{tabular}

En cuanto a la diversidad de opiniones, el ítem que tiene mayor homogeneidad es el 3.2 "La organización permite una búsqueda selectiva distinguiendo entre grupos temáticos, etapas, dificultades..." ( $S D=, 580)$. Una vez más, este ítem aparece, por lo que se afianza positivamente la opinión de esta afirmación. El ítem 3.9 "Soy informado frecuentemente sobre los nuevos recursos ofrecidos por la Consejería de Educación" $(S D=, 900)$ es el que ha obtenido mayor heterogeneidad, es decir, hay opiniones de todo tipo. Además, este ítem ha tenido el valor mínimo de uno.

Utilidad/adecuación de los recursos 
Podemos observar en la Tabla 5 que los orientadores han otorgado una valoración más positiva al ítem 4.3 "La información que aporta es útil para mi trabajo" $(M=4,20)$ Sin embargo, el ítem 4.7 "Reenvío a mis compañeros para la búsqueda de recursos y/o información" $(M=3,36)$ ha obtenido la media más baja en esta dimensión.

Tabla 5.

Utilidad/adecuación de los recursos desde la opinión de los orientadores. Estadística descriptiva

\begin{tabular}{|l|c|c|c|c|}
\hline \multicolumn{1}{|c|}{ Ítems utilidad/adecuación de los recursos } & M & SD & Mín. & Máx. \\
\hline 4.1 Consulto habitualmente ORIENTAMUR. & 4,18 &, 834 & 1 & 5 \\
\hline $\begin{array}{l}\text { 4.2 Cumple mis pretensiones como profesional de la } \\
\text { Orientación. }\end{array}$ & 3,91 &, 821 & 2 & 5 \\
\hline $\begin{array}{l}\text { 4.3 La información que aporta es útil para mi } \\
\text { trabajo. }\end{array}$ & 4,20 &, 786 & 2 & 5 \\
\hline 4.4 La normativa está actualizada. & 3,91 & 1,019 & 1 & 5 \\
\hline $\begin{array}{l}\text { 4.5 La bibliografía está específicamente relacionada } \\
\text { a temas/bloques temáticos concretos. }\end{array}$ & 3,91 &, 925 & 2 & 5 \\
\hline $\begin{array}{l}\text { 4.6 Los recursos respetan la normativa } \\
\text { correspondiente a derechos de autor y de propiedad } \\
\text { intelectual. }\end{array}$ & 4,16 &, 645 & 2 & 5 \\
\hline $\begin{array}{l}\text { 4.7 Reenvío a mis compañeros para la búsqueda de } \\
\text { recursos y/o información. }\end{array}$ & 3,36 & 1,131 & 1 & 5 \\
\hline $\begin{array}{l}4.8 \text { Los recursos destinados a los orientadores son } \\
\text { adecuados. }\end{array}$ & 4,11 &, 775 & 2 & 5 \\
\hline $\begin{array}{l}4.9 \text { Los recursos orientados a las familias son } \\
\text { adecuados a sus necesidades. }\end{array}$ & 3,56 &, 755 & 2 & 5 \\
\hline $\begin{array}{l}\text { 4.10 Los recursos orientados a los alumnos son } \\
\text { adecuados a sus necesidades. }\end{array}$ & 3,58 &, 812 & 2 & 5 \\
\hline $\begin{array}{l}\text { 4.11 Los recursos orientados al profesorado son } \\
\text { adecuados a sus necesidades. }\end{array}$ & 3,60 &, 720 & 2 & 5 \\
\hline $\begin{array}{l}4.12 \text { Los recursos orientados a los tutores son } \\
\text { adecuados a sus necesidades. }\end{array}$ & 3,62 &, 834 & 2 & 5 \\
\hline
\end{tabular}

Pero no todos los orientadores opinan de la misma manera, este último ítem, 4.7 "Reenvío a mis compañeros para la búsqueda de recursos y/o información" ( $S D=1,131)$, ha obtenido la mayor disparidad de opiniones. El ítem 4.6 "Los recursos respetan la normativa correspondiente a derechos de autor y de propiedad intelectual" $(S D=, 645)$ ha sido el que ha tenido una respuesta más homogénea con un grado de acuerdo alto $(M=4,16)$.

\section{Aspectos pedagógicos}

En la Tabla 6 podemos resaltar que, las opiniones de los orientadores no han sido demasiado favorables, especialmente en el ítem 5.2 "Utilizo el apartado de contacto para resolver preguntas frecuentes" $(M=2,91)$. La opinión valorada más favorablemente ha sido el ítem 5.1 "Los recursos presentan una información actual e innovadora" $(M=3,80)$. 
Tabla 6.

Aspectos pedagógicos, sobre el portal ORIENTAMUR, en opinión de los orientadores. Estadística descriptiva

\begin{tabular}{|l|c|c|c|c|}
\hline \multicolumn{1}{|c|}{ Ítems aspectos pedagógicos } & M & SD & Mín. & Máx. \\
\hline $\begin{array}{l}5.1 \text { Los recursos presentan una información actual e } \\
\text { innovadora. }\end{array}$ & 3,80 &, 919 & 1 & 5 \\
\hline $\begin{array}{l}5.2 \text { Utilizo el apartado de contacto para resolver } \\
\text { preguntas frecuentes }\end{array}$ & 2,91 & 1,203 & 1 & 5 \\
\hline $\begin{array}{l}5.3 \text { El portal permite contribuciones por parte de los } \\
\text { orientadores. }\end{array}$ & 3,36 & 1,351 & 1 & 5 \\
\hline
\end{tabular}

Si revisamos la diversidad de opiniones, nos damos cuenta de que en todos los ítems hay una gran discrepancia, destacando el 5.3 "El portal permite contribuciones por parte de los orientadores" $(S D=1,351)$.

\section{Motivación e interés de los recursos}

Realizando un análisis de la Tabla 7 podemos apreciar el mayor grado de acuerdo en el ítem 6.5 "Difundo entre mis compañeros los recursos disponibles" $(M=4,00)$. La opinión menos satisfactoria viene de la mano del ítem 6.1 "Son motivadores como orientador de Educación Secundaria" $(M=3,47)$.

Tabla 7.

Motivación e interés de los recursos desde la opinión de los orientadores. Estadística descriptiva

\begin{tabular}{|l|c|c|c|c|}
\hline \multicolumn{1}{|c|}{ Ítems motivación e interés de los recursos } & M & SD & Mín. & Máx. \\
\hline $\begin{array}{l}\text { 6.1 Son motivadores como orientador de Educación } \\
\text { Secundaria. }\end{array}$ & 3,47 & 1,531 & 1 & 5 \\
\hline 6.2 Los recursos imprimibles son suficientes. & 3,91 &, 821 & 2 & 5 \\
\hline 6.3 Los recursos interactivos son suficientes. & 3,49 &, 968 & 2 & 5 \\
\hline $\begin{array}{l}\text { 6.4 Los recursos educativos ayudan a mejorar mi } \\
\text { trabajo. }\end{array}$ & 3,40 &, 915 & 1 & 5 \\
\hline $\begin{array}{l}\text { 6.5 Difundo entre mis compañeros los recursos } \\
\text { disponibles }\end{array}$ & 4,00 &, 769 & 2 & 5 \\
\hline
\end{tabular}

La mayor diversidad de opiniones de todos los ámbitos se encuentra en el ítem 6.1 "Los recursos son motivadores como orientador de Educación Secundaria" ( $S D=1,531)$. El ítem con mayor homogeneidad es el 6.5 "Difundo entre mis compañeros los recursos disponibles" ( $S D=, 769)$. 


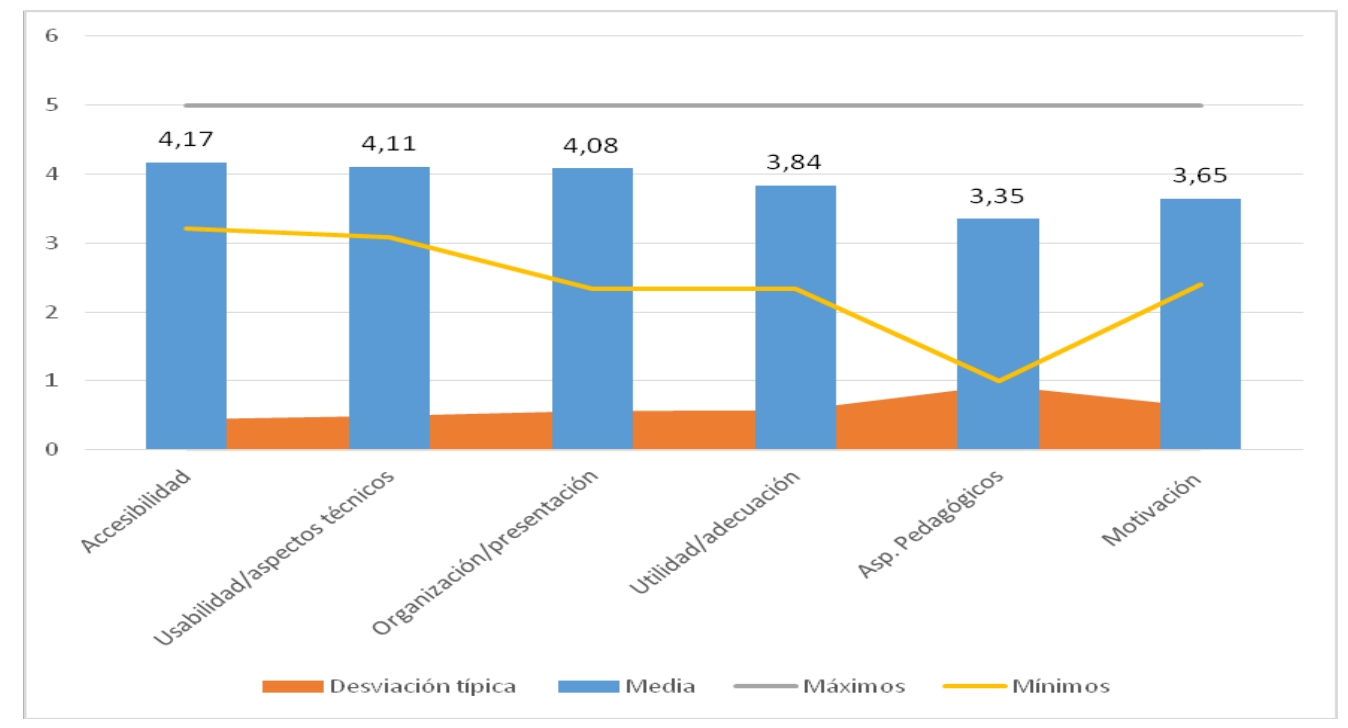

Figura 1: Valoración media de las dimensiones del cuestionario en opinión de los orientadores. Estadística descriptiva.

Como podemos ver en la Figura 1, las tres primeras dimensiones obtienen el mayor grado de acuerdo, es decir, los orientadores tiene una opinión formada más positiva para las siguientes dimensiones: accesibilidad al portal de Atención a la Diversidad: ORIENTAMUR, usabilidad/aspectos técnicos y organización y presentación de los recursos. El aspecto pedagógico ha sido en el que ha obtenido un mayor grado de desacuerdo, presentando la media más baja $(M=3,35)$.

Con respecto a la diversidad de opiniones, podemos observar que existe una clara heterogeneidad en el aspecto pedagógico, existiendo una mayor homogeneidad en las demás dimensiones.

Con estos resultados, podemos mencionar que, según los orientadores que han colaborado con este proyecto de investigación, las dos primeras dimensiones, de acceso, usabilidad/aspectos técnicos son las que han obtenido un grado de mayor acuerdo

Para terminar el apartado de análisis descriptivo, revisaremos la valoración global del portal ORIENTAMUR, como siempre, desde la perspectiva de los orientadores. Podemos ver los datos extraídos en la Tabla 8.

Tabla8.

Valoración global del portal de Atención a la Diversidad ORIENTAMUR desde la perspectiva de los orientadores. Estadística descriptiva 


\begin{tabular}{|l|c|c|c|c|}
\hline & M & SD & Mín. & Máx. \\
\hline Valoración global & 3,8 &, 44 & 2,81 & 4,76 \\
\hline
\end{tabular}

\section{Análisis de contraste de medias en función de variables sociodemográficas}

A continuación, realizaremos un análisis comparativo de las opiniones de los orientadores según las variables: titulación, edad, sexo, experiencia docente, grado de formación en TIC, situación laboral y tipo de centro. Para ello utilizaremos dos pruebas principalmente, la prueba $\mathrm{H}$ de Kruskal - Wallis y la prueba U de Mann Whitney.

Los resultados, tras realizar la prueba de $\mathrm{H}$ de Kruskal - Wallis, nos indican que no existe ninguna diferencia significativa, en función de la variable titulación. Si bien en cuanto a la variable edad, en la Tabla 9, apreciamos que existen diferencias estadísticamente significativas respecto a la opinión de los orientadores en la dimensión de motivación e interés de los recursos.

Tabla 9.

Prueba de contraste de medias en cada dimensión en función de la edad de los orientadores

\begin{tabular}{|l|c|}
\hline \multicolumn{1}{|c|}{ Dimensiones } & $\boldsymbol{p}$ \\
\hline Accesibilidad al portal ORIENTAMUR &, 279 \\
\hline Usabilidad/aspectos técnicos &, 377 \\
\hline Organización y presentación de los recursos &, 193 \\
\hline Utilización y adecuación de los recursos &, 063 \\
\hline Aspectos pedagógicos &, 741 \\
\hline Motivación e interés de los recursos &, $033^{*}$ \\
\hline Media global &, 065 \\
\hline
\end{tabular}

Para ahondar en el proceso, hemos realizado la prueba de $U$ de Mann Whitney con el objetivo de localizar las franjas de edad entre las que existirían diferencias estadísticamente significativas, en relación a la edad de los orientadores; encontrando diferencias estadísticamente significativas significativa entre los sujetos con una franja de edad entre 36 y 45 años y los de más de 55 años $(p=, 005)$. Dicha diferencia es favorable a los orientadores de 36 a 45 años, opinando que los recursos de este portal les motivan más en su trabajo que los orientadores de más de 55 años.

En cuanto a la variable sexo, se aprecian diferencias estadísticamente significativas en la Tabla 10 en función del sexo de los orientadores, respecto a su valoración media global y en todas las dimensiones de valoración del portal ORIENTAMUR. En todos los casos las diferencias se producen a favor de las mujeres. 
Tabla 10.

Prueba de contraste de medias en cada dimensión en función del sexo de los orientadores

\begin{tabular}{|l|l|}
\hline \multicolumn{1}{|c|}{ Dimensiones } & $\boldsymbol{p}$ \\
\hline Accesibilidad al portal ORIENTAMUR &, $007^{*}$ \\
\hline Usabilidad/aspectos técnicos &, $029^{*}$ \\
\hline Organización y presentación de los recursos &, $109^{*}$ \\
\hline Utilización y adecuación de los recursos &, $013^{*}$ \\
\hline Aspectos pedagógicos &, $242^{*}$ \\
\hline Motivación e interés de los recursos &, $064^{*}$ \\
\hline Media global &, $006^{*}$ \\
\hline
\end{tabular}

Respecto a la influencia de la experiencia docente en las opiniones de los orientadores, los resultados nos han mostrado que no existen diferencias estadísticamente significativas.

El grado de formación en TIC, los resultados de la Tabla 11, encontramos diferencias estadísticamente significativa entre el ámbito de motivación e interés $(p=, 028)$ y la media global $(p=, 022)$.

Tabla 11.

Prueba de contraste de medias en cada dimensión en función del grado de formación en TIC de los orientadores

\begin{tabular}{|l|c|}
\hline \multicolumn{1}{|c|}{ Dimensiones } & $\boldsymbol{p}$ \\
\hline Accesibilidad al portal ORIENTAMUR &, 191 \\
\hline Usabilidad/aspectos técnicos &, 417 \\
\hline Organización y presentación de los recursos &, 225 \\
\hline Utilización y adecuación de los recursos &, 091 \\
\hline Aspectos pedagógicos &, $051^{*}$ \\
\hline Motivación e interés de los recursos &, $028^{*}$ \\
\hline Media global &, $022^{*}$ \\
\hline
\end{tabular}

Además encontramos un dato parcialmente significativo, la dimensión de aspectos pedagógicos. Realizando posteriormente la prueba $U$ de Mann Whitney podemos profundizar en estos resultados quedando reflejados en la Tabla 12. 
Tabla 12.

Prueba de contraste de medias (Mann Whitney) en las dimensiones de aspectos pedagógicos, motivación e interés y media global, en función del nivel de formación en TIC de los orientadores

\begin{tabular}{|c|c|c|c|c|c|c|c|c|}
\hline \multicolumn{3}{|c|}{ Aspectos pedagógicos } & \multicolumn{3}{c|}{ Motivación e interés } & \multicolumn{3}{c|}{ Media global } \\
\hline $\begin{array}{c}\text { Grado } \\
\text { TIC }\end{array}$ & $p$ & Grado TIC & $\begin{array}{c}\text { Grado } \\
\text { TIC }\end{array}$ & $p$ & Grado TIC & $\begin{array}{c}\text { Grado } \\
\text { TIC }\end{array}$ & $p$ & Grado TIC \\
\hline básico &, $043^{*}$ & avanzado & básico &, $043^{*}$ & avanzado & básico &, $031^{*}$ & avanzado \\
\hline básico &, 627 & usuario & básico &, 206 & usuario & básico &, 454 & usuario \\
\hline usuario &, $027^{*}$ & avanzado & usuario &, $024^{*}$ & avanzado & usuario &, $012^{*}$ & avanzado \\
\hline
\end{tabular}

Si centramos nuestra atención en la Tabla 12, encontramos varias diferencias estadísticamente significativas: entre el nivel básico y avanzado, y usuario y avanzado de cada una de las dimensiones; tanto en motivación, aspectos pedagógicos como en la media global. Así, dependiendo del nivel de formación en TIC de los orientadores valoran de manera diferente el portal.

La variable situación laboral de cada orientador, si es interino, funcionario o contratado, también se ha analizado en este estudio. Tras realizar la prueba $\mathrm{H}$ de Kruskal - Wallis no observamos ningún dato estadísticamente significativo.

Por último, analizando la variable tipo de centro (público, privado concertado y privado) en el que trabajan los orientadores participantes observamos que no existe ninguna diferencia significativa.

\section{Observaciones y propuestas de mejora en opinión de los orientadores}

Al completar la encuesta, el orientador cuenta con un espacio para observaciones/sugerencias y propuestas de mejora. Entre las respuestas dadas por este profesional en este espacio, hay una que es reiterada y es la necesidad de una actualización. Además, también propone que toda la legislación y documentos anexos que necesite el orientador en su labor se encuentren recogidos en este portal.

\section{Discusión y conclusiones}

Los resultados muestran que la valoración global del portal ORIENTAMUR, según la opinión de los orientadores, ha sido positiva, con una nota de notable, como hemos explicado anteriormente, encontrando en las respuestas, en general, un alto nivel de homogeneidad.

Este portal destaca fundamentalmente en tres dimensiones desde la percepción de los orientadores, en este orden: accesibilidad al portal de Atención a la Diversidad: ORIENTAMUR, usabilidad/aspectos técnicos y organización y presentación de los recursos (todas con una media superior a cuatro puntos de un total de cinco). Dentro de estas dimensiones encontramos algunos puntos fuertes a destacar, por ejemplo, el acceso permanente que tienen los orientadores desde su departamento al portal ORIENTAMUR, que el texto es legible en todo el sitio web y que este portal permite una búsqueda selectiva distinguiendo entre grupos temáticos etapas, dificultades... Sin embargo, también pueden mejorarse ciertos aspectos que han sido los menos apreciados por los orientadores participantes como el ítem 1.6 "La interfaz de usuario cuenta con opciones de accesibilidad para personas con discapacidad", lo que nos puede indicar que los orientadores desconozcan este servicio y/o nunca lo hayan utilizado.

El aspecto pedagógico ha sido la dimensión menos valorada por los orientadores. Además, es la dimensión en la que hemos obtenido respuestas más dispares. Podemos deducir de 
esta estimación que una posible mejora futura del portal podría devenir de este aspecto. Podría mejorarse la interactividad y el feedback entre los orientadores y el Servicio de Atención a la Diversidad de ORIENTAMUR; ya que hemos observado que los orientadores no se suelen poner en contacto para resolver preguntas frecuentes. Además, los orientadores opinan que los recursos que proceden de las buenas prácticas del profesorado que contiene el portal no son suficientes por lo que promover este tipo de acciones podría ser un impulso positivo a este portal.

Hemos encontrado que las respuestas han sido independientes de variables como la titulación del orientador, la experiencia docente, la situación laboral y el tipo de centro. Sin embargo, la opinión de los orientadores sí que depende de factores como la edad, en concreto entre los orientadores de 36 a 45 años y los de más de 55 años. Los orientadores cuya edad se sitúa en una franja intermedia opinan que los recursos del portal les motivan más en su trabajo que los orientadores mayores de 55 años. Esto puede deberse a un mayor manejo de las TIC y de búsqueda en el portal que los orientadores con una edad mayor. En cuanto al sexo, las mujeres muestran un grado de acuerdo significativamente mayor respecto a los hombres en la valoración global del portal ORIENTAMUR y en todos los demás aspectos evaluados: accesibilidad, usabilidad y aspectos técnicos, organización y presentación, aspectos pedagógicos y motivación en su uso. Esto puede deberse a que estén más familiarizadas con la página haciendo más uso de ella que los hombres. Por último, también hemos encontrado diferencias significativas en cuanto al grado de formación en TIC, encontrándose ésta entre los orientadores que opinan que poseen un nivel básico y los que afirman tener un nivel avanzado. Éstos últimos son más críticos con la página mientras que los primeros la valoran de una forma más favorable.

Por otro lado, no debemos olvidar las observaciones realizadas por los orientadores participantes concretadas en la necesidad de una actualización del portal. Puede que sea el momento de introducir una mayor interacción e interactividad en ORIENTAMUR como he aclarado anteriormente. También podemos solicitar mayor originalidad y creatividad de los recursos prácticos y que éstos sean motivadores para la labor del orientador.

Hemos podido comprobar los estudios de Carballo et al. (1997) y Vélaz-de-Medrano et al. (2012) en los que nos señalaban que la falta de recursos era, entre otros factores, uno de los principales obstáculos o dificultades de un orientador. En nuestra encuesta, más del $50 \%$ de los orientadores opinan que los recursos disponibles en el portal ORIENTAMUR son suficientes por lo que podemos apuntar que, o bien no se produce ninguna escasez de recursos en los centros educativos de Educación Secundaria de esta región o que, aunque exista esta escasez, no proviene del portal para la atención a la diversidad siendo éste suficientemente completo. Por otro lado, debemos añadir que todos los orientadores consideran que tienen el conocimiento necesario para utilizar los recursos. Sin embargo, lo que sí proponen los orientadores como mejora del portal sería la mayor actualización de algunos de los aspectos o temáticas que se pueden consultar a través del mismo, lo que facilitaría la labor de asesoramiento psicopedagógico de este profesional y le garantizaría un mayor nivel de calidad, al disponer de la información más actualizada de su ámbito profesional de intervención. 


\section{Referencias}

Aciego, R., Álvarez, P. \& Muñoz, M.C. (2005). Análisis del rol profesional del asesor psicopedagógico: una visión desde la práctica. Revista Cultura y Educación, 17, 35-52.

Álvarez, P., Bethencourt, J. \& Cabrera, L. (2000). La transición al mercado laboral de los psicopedagogos: estudio de las dos primeras promociones en la Universidad de La Laguna. Revista de Psicología General y Aplicada. 53(3), 553-547. Extraído de: https://www.google.es/url?sa=t\&rct=j\&q=\&esrc=s\&source=web\&cd=1\&cad=rja\&uac $\mathrm{t}=8$ \&ved $=0 \mathrm{CCIQFjAA \& url=http} \% 3 \mathrm{~A} \% 2 \mathrm{~F} \% 2 \mathrm{Fdialnet}$.unirioja.es\%2Fdescarga\%2Farticulo\% 2F2357090.pdf\&ei=t6GVda6OsjiUYqykbgK\&usg=AFQjCNEJoRsDYCgHUPXT2HMZKbHKJPByg\&sig2=PKkEwE9qjw-Q22izW48dUA[revisado 16 febrero 2015]

Arnáiz, P. \& Guirao, J. M. (2015). La autoevaluación de centros en España para la atención a la diversidad desde una perspectiva inclusiva: ACADI. Revista Electrónica Interuniversitaria de Formación del Profesorado, 18(1), 45-101.

Cabero, J. \& Duarte, A. (2000). Evaluación de medios y materiales de enseñanza en soporte multimedia. Comunicación y Pedagogía: Nuevas Tecnologías y Recursos Didácticos,166, 17-28.

Couper, P. \& Miller, P. (2008). Web survey methods. Introduction. Public Opinion Quarterly.72(5), 831835.

Comes, G., Parera, B., Vedriel, G. \& Vives, M. (2009). El psicopedagogo y la psicopedagoga en Cataluña. Revista de Ciènces de l' Educació, III época, 225-239.

Cano, M., Mayoral, P., Liesa, E. \& Castelló, M. (2013). Valoración de las funciones del profesor de orientación educativa en Cataluña. Revista Española de Orientación y Psicopedagogía (REOP), 24(3), 80-97.

Carballo, R., Fernández, M.J., Díaz, M.T \& García, N. (1997). Orientación en Educación Secundaria: situación actual y prospectiva. Revista de Investigación educativa. 15(2), 9-83.

De Vellis, R.F. (2003). Scale development: Theory and applications ( $2^{\mathrm{a}}$ ed.). Thousand Oaks, CA: Sage.

Escarbajal, A., Mirete,, A.B., Maquilón,„J. J., Izquierdo, T., López Hidalgo, J. I., Orcajada, N.\& Sánchez Martín, M. (2012). La atención a la diversidad: la educación inclusiva. REIFOP, 15(1), 135-144. Extraído en: http//www.aufop.com

Hernández Pina, F.\&Maquilón, J.J. (2010). Introducción a los diseños de investigación educativa». En S. Nieto (Ed.) Principios, métodos y técnicas esenciales para la investigación educativa (pp. 109-126). Madrid: Dykinson.

Ley Orgánica 2/2006, de 3 de mayo, de Educación.

Ley Orgánica 8/2013 de 9 de noviembre para la Mejora de la Calidad Educativa. 
Liesa, E., Castelló, M., Carretero, R., Cano, M. \& Mayoral, P. (2012). La atención a la diversidad en la Educación Secundaria Obligatoria: valoraciones de los protagonistas. Profesorado: Revista de Currículum y formación del profesorado, 16(2), 451-468.

Marcelo, C. \& Zapata, M. (2008). Cuestionario para la evaluación: "evaluación de la calidad para programas completos de formación docente a través de estrategias de aprendizaje abierto y a distancia". Metodología de uso y descripción de indicadores. RED: Revista de Educación a Distancia. Extra $n^{\circ}$ 7, (Ejemplar dedicado a: Evaluación de la calidad en entornos virtuales de aprendizaje).

Marqués, P. (1998).Criterios para la clasificación y evaluación de espacios web de interés educativo. Educar: revista de la Secció de Ciencies de l'Educació, 25, 95-111.

Mirete, A. B., Cabello, F., Martínez-Segura, M. J. \& García-Sánchez, F. A. (2013). Cuestionario para la evaluación de aspectos didácticos, técnicos y pedagógicos de webs didácticas. Murcia: EDITUM. Extraído de: http://www.um.es/webs_didacticas/instrumentos/CEETP/CEETP_files/CEETP\%2oversi on\%20extendida.pdf [revisado 18 febrero 2015]

Vélaz-de-Medrano, C., Repetto, E., Blanco, A., Galán, A., Guillamón, J.R., Negro, N. \& Torrego, J.C. (2001). El desarrollo profesional de los orientadores de Educación Secundaria: análisis y prospectiva. Revista de investigación educativa, 19, 199-220.

Vélaz-de-Medrano, C., Manzano, S. \& Blanco, A. (2012). Los procesos de cambio de las políticas públicas sobre orientación y apoyo a la escuela: análisis comparado de sistemas vigentes y emergentes. Estudio múltiple de casos en una muestra de Comunidades Autónomas. Madrid: Instituto de Formación del Profesorado, Investigación e Innovación Educativa (IFIIE). Extraído de: http://www.uned.es/grisop/documentos/Doc\%208.pdf 